\title{
Experiences of Adolescents Seeking Multidisciplinary Treatment for Chronic Pain
}

\author{
Katie Fleischman ${ }^{1}$, Ling-LunChien ${ }^{1}$, Anthony A. Hains ${ }^{1, *}$, Heidi F. Christianson ${ }^{2}$, Renee J. Ladwig ${ }^{3}$, \\ Kimberly A. Khan ${ }^{2,3}$, Hobart Davies ${ }^{1}$, Keri R. Hainsworth ${ }^{2}$, Molly M. Garwood ${ }^{3}$, Steven J. Weisman ${ }^{2,3}$ \\ ${ }^{1}$ University of Wisconsin-Milwaukee. P.O. Box 4132200 E. Kenwood Blvd, Milwaukee WI 53201-0413 \\ ${ }^{2}$ Medical College of Wisconsin,8700 W. Wisconsin Avenue, Milwaukee WI 53226 \\ ${ }^{3}$ Children's Hospital of Wisconsin, 9000 Wisconsin Avenue, Milwaukee, WI 53226 \\ *Corresponding Author: aahains@uwm.edu
}

Copyright $@ 2014$ Horizon Research Publishing All rights reserved.

\begin{abstract}
Twelve adolescents receiving treatment from a hospital- based pain clinic were interviewed about their experience of receiving an initial multidisciplinary evaluation for chronic pain. Using Consensual Qualitative Research (CQR), categories and subcategories were identified based on adolescents' perception of their initial appointment with the multidisciplinary treatment team. Findings suggest that exposure to multiple treatment options may have led to variability in the adolescents' level of satisfaction/dissatisfaction with certain aspects of their care. Implications for practice and research are discussed.
\end{abstract}

Keywords Qualitative Research, Adolescents, Chronic Pain, Multidisciplinary Care

\section{Introduction}

Teenagers with chronic pain, as well as their parents, have several encounters with health care providers throughout the treatment of their pain. Medical professionals perform a myriad of diagnostic tests, review physiological explanations for pain, and give differing opinions regarding the source and treatment of the distress. Coping with and navigating the treatments for chronic pain can be a difficult process, burdening adolescents, their parents, and families. Many pain treatment providers have shifted toward a multidisciplinary approach for the treatment of pediatric chronic pain to treat physical, psychological, and social aspects of the patient and his/her family. Although a few studies have assessed the effectiveness of these approaches[1, $2,3,4]$ little is known about how the adolescent experiences such an approach. Previous quantitative research has found significant improvement in both pain symptoms and functional ability after multidisciplinary pain treatment $[1,2$, 4]. Even though research has proposed that this approach can be more effective than specialists consulting family physicians [2] little is known about why the approach works or how adolescents react to such approaches. Consequently, the "initial intake experience" as perceived by adolescents living with chronic pain and their parents must be revealed, to better comprehend the breadth of factors that impact the modern standard of care for this population.

Little is known about the experience of receiving multidisciplinary treatment for chronic pain, especially regarding expectations prior to treatment, changes in expectations throughout the treatment, and comparisons with other forms of treatment. Since adolescents have likely had multiple previous encounters with the medical establishment, an understanding of how adolescents make sense of multidisciplinary pain treatment is essential to understanding how to effectively and appropriately address this population's needs.

The purpose of this study was to explore the perspective of adolescent pain patients' regarding the initial experience with a multidisciplinary pain treatment team. Specifically, this study addressed the expectations of treatment prior to the intake session with a multidisciplinary team, how those expectations changed throughout the session, reactions after the session, and finally comparisons to other treatments for chronic pain. Although a few studies have found multidisciplinary treatment approaches for chronic pain to be effective [1-4], it is critical to strive for improvements in any treatment approach. Therefore, it is important to understand the patient's perspective on the process. A gap in the literature exists with regard to the manner in which adolescents and their parents react to the initial intervention. A qualitative study affords the unique opportunity to thoroughly evaluate such reactions, and in turn, to improve treatment approaches. By examining the first hand experiences of adolescents receiving an initial multidisciplinary evaluation for chronic pain, health care providers may gain a more comprehensive understanding of how the initial appointment impacts patients and their families. In turn, initial interactions may be improved, 
ultimately resulting in better treatment outcomes.

\section{Method}

\subsection{Design}

In order to capture and explore the adolescents' experience, a qualitative design based on Consensual Qualitative Research (CQR) was used [6]. The CQR methodology allowed us to describe in depth the adolescents' expectations and experiences with a multidisciplinary pain team by capturing the phenomena as it naturally occurred through the use of open ended questions. Due to the qualitative nature of the project, no specific hypotheses were developed; rather conclusions were derived from the adolescents' responses to the questions. Participants were viewed as the experts of their experience and researchers drew conclusions about the adolescents' experiences based on findings across cases. Therefore, pain conditions, reported pain levels, or medication usage were not controlled. Rather, the goal of the study was to capture the participants' whole experience. The emphasis $\mathrm{CQR}$ placed on using multiple researchers to form a consensus on decisions, verify results, and to discuss biases throughout the study's completion, demonstrates the method's scientific rigor [6].

\subsection{Participants}

Participants were twelve adolescents with chronic pain ranging in age from 12 to 17 years old $(\mathrm{M}=14.75$ years old; $\mathrm{SD}=1.712)$ and their duration of pain ranged from 1 to 60 months $(\mathrm{M}=14$ months; $\mathrm{SD}=15.879)$. The number of participants was consistent with CQR methodology which suggests using a sample size of 8 to 15 participants. Adolescents were outpatients receiving multidisciplinary treatment from a local hospital pain clinic and were recruited for the study after their initial intake session. No youth were excluded from the study based on gender, race, or ethnicity.

\subsection{Researchers}

The primary team consisted of three counseling psychology doctoral students. All members read articles and held several discussions on consensual qualitative research (CQR) and qualitative interviewing. A professor from the counseling psychology program served as the auditor. The professor and one of the doctoral students had prior experience researching psychosocial implications in children with chronic pain. Before the data collection, members discussed their expectations and biases. The primary team held expectations based on literature reviews and previous research experience that adolescents' may have varied expectations and experiences when meeting with the multidisciplinary treatment team for the first time.

\subsection{Participant Selection}

Data was collected from twelve adolescents that were seen as outpatients in the pain clinic of a pediatric hospital in a large mid-western city for the management and treatment for their chronic pain. Members of the multidisciplinary pain treatment team gave a brief written description of the study to the adolescents and their accompanied parents during the initial appointment. Parents and adolescents who were interested signed consent and assent forms, respectively, and an appointment was scheduled for the interview to be conducted in the family's home within one week of the initial intake with the multidisciplinary team. This study was reviewed and approved by the university's and local hospital's IRB committee.

\subsection{Multidisciplinary Chronic Pain Clinic}

The initial interdisciplinary pain clinic evaluation consists of a $1 \frac{1}{2}$ hour visit where the adolescent and family meet with a physician, psychologist or family therapist. During the evaluation with the adolescent and family members, medical history and general functioning information are collected. Next, the physician completes a physical assessment with the adolescent while the mental health practitioner interviews the parents. If appropriate, a physical therapist will also conduct a physical assessment of the adolescent. Then, the mental health practitioner will interview the adolescent independently. After the initial assessment is complete, the physician and mental health practitioner meet to develop a treatment plan followed by a final presentation of the treatment plan to the family. The initial interdisciplinary pain clinic evaluation includes mindful attention to the adolescents' and families' beliefs and expectations, as the practitioners focus on validating and acknowledging their challenges and strengths. A primary focus of the initial appointment is to develop a shared rationale for understanding the adolescent's and families' distress, testing their response to reframing irrational beliefs, providing a rationale for treatment recommendations, and developing a comprehensive treatment plan. Each treatment plan is individually tailored and may include medication management, physical therapy, individual and family cognitive behavioral therapy, school accommodation and return to school plan, collaboration with existing medical and mental health practitioners and school staff, and pain team follow-up appointments.

\subsection{Interview Protocol}

Researchers from the Counseling and Clinical Psychology program developed the interview protocol based on a literature review related to adolescent chronic pain and multidisciplinary pain treatment, and based on consultation with a local multidisciplinary pain clinic team. As part of this process, the researchers examined and openly discussed any biases in an effort to avoid tainting the interview protocol or the various steps of the data analysis. The interview guide included demographic data and open-ended topics such as 
expectations and experiences throughout the first clinic appointment, description of how feelings changed throughout the appointment, thoughts about the treatment after leaving the appointment, and contrast with previous medical care. Interview questions were open-ended to allow participants considerable freedom in their responses and to avoid leading or polarizing questions.

Members from the counseling and clinical psychology research teams and researchers from the medical college completed twelve interviews. Recorded, face to face interviews were conducted. The initial meeting with the adolescent took place at their residence to ensure comfort and confidentiality. The researchers reviewed the length of the interview (approximately one hour), the topics covered, confidentiality and limits thereof, and compensation. After each adolescent and one of their guardians were consented, the researcher and adolescent met in a quiet area for taping and privacy purposes. All questions were asked to each adolescent and lists of probes were used to gather any additional information or clarification from the adolescent on these issues. Furthermore, additional probes were used to explore issues that emerged in the interview for each participant [6].

\subsection{Transcripts}

All interviews were taped and then transcribed verbatim. Numbers were assigned to the recordings and transcriptions, and no names were written on the transcripts. As soon as the transcripts were completed, all audiotapes were destroyed.

\subsection{Data Analysis}

Following the procedures by Hill[6], three main steps were utilized for analyzing the data. First, domains were developed by segmenting the adolescents' responses into themes. Second, core ideas were created by concisely summarizing, without interpretation, for each adolescent's response from each domain. Third, cross analysis was constructed by discovering common themes within each domain that derived from the core ideas across cases. During each stage of analysis, the primary team independently analyzed the data, then met as a team in order to reach consensus on a unified decision that best represented the data. Finally, the auditor reviewed the decisions at each stage of the analysis to make sure the results accurately reflected the data. At that point, the primary team had the decision to accept or reject any of the auditor's suggestions [8].

\section{Results}

The following six domains were constructed from the interviews: Experience with Past History, Technical History, Expectation of Treatment, General Expectation, Family Involvement, and General Experience (See Table 1).
Following the procedure described by Hill [6] categories were developed from core ideas across all adolescents within each domain. CQR methodology recommends illustrating the results within a table to show how frequently categories apply to the entire sample. Categories were considered "General" in nature when all or all but one of the adolescents reported the same issue (10-12 cases in the current study), "Typical" when 7-9 adolescents reported the same issue; and "Variant" when 4-6 reported the issue. Categories that only apply across one to three categories were considered "Rare," and were dropped due to not being descriptive of the data.

\subsection{Experience with Previous Providers}

Generally, adolescents identified their experience with previous providers as dissatisfying. Adolescents reported feeling discouraged and uncomfortable with their previous providers approach to treatment. For example, one adolescent stated, “The other treatment team...I really didn't feel comfortable talking with them and I didn't want to talk to them, because, a lot of them....were in a rush and stuff...they would come in and out." Another adolescent explained, "They would have tried if they can. I've been going through this for a couple of months, so if they could have fixed it...it would have been fixed."

Adolescents who typically reported a neutral experience, noted differences between their previous providers and the multidisciplinary team, or could not recall a specific experience with their past providers. For instance, one adolescent exclaimed, "I don't really remember that much...I just forget all about them." While another adolescent reported, "Yeah, and he [past provider] basically, he treated adults more than pediatrics...he wasn't a pediatric doctor at all."

On the other hand, adolescents were also variantly satisfied with their previous care. Adolescents noted that their previous medical team was helpful and they felt confident and comfortable with their approach.

\subsection{Treatment History}

Adolescents generally stated they were dissatisfied with their medication management from previous providers. Adolescents reported their previously prescribed medication was not meeting their pain needs and expressed interest in alternate medication choices. They also noted feeling like they were on too much medication and did not like the side effects. One adolescent explained, "We pretty much talked to his nurse and they said, "Just Advil, we can't, I don't think we can give you anything else, so we didn't get...didn't really get what I needed from them." Another adolescent reported, "They had a bag of medications. It was about 5 or 7 things they had me go on and it was way too much. Most of it wasn't helping and they were just guessing...nobody really knew what it really was and that really frustrated me." 
Table 1. Domains and categories of adolescents' perceptions of multidisciplinary pain treatment

\begin{tabular}{|c|c|c|}
\hline Domain & Category & Frequency of Practitioner Reports \\
\hline \multirow{3}{*}{ Experience with past history } & Unsatisfied with previous care & Typical \\
\hline & Neutral experience with previous care & Typical \\
\hline & Satisfied with previous care & Variant \\
\hline \multirow{5}{*}{ Technical History } & Prior treatment was unhelpful & General \\
\hline & Poor medication management & General \\
\hline & Prior treatment helpful & Typical \\
\hline & Treatment orientated/less communication with youth & Typical \\
\hline & Dealt with only certain aspects of pain & Variant \\
\hline \multirow{3}{*}{ Expectation of Treatment } & Expected to communicate with team & Typical \\
\hline & Predicted medication management & Variant \\
\hline & Apprehension to treatment & Variant \\
\hline \multirow{6}{*}{ General Expectation } & Anticipatory nervousness/anxiety & Typical \\
\hline & No identified expectations or emotions, feeling neutral & Typical \\
\hline & Predicted similar experience to other physicians & Variant \\
\hline & Didn't know what to expect & Variant \\
\hline & Expected multiple doctors and length of appointment & Variant \\
\hline & Negative view of treatment & Variant \\
\hline \multirow{3}{*}{ Family Involvement } & Parental/guardian lead communication & General \\
\hline & Parental support to youth & Typical \\
\hline & Families hopefulness for treatment & Typical \\
\hline \multirow{12}{*}{ General Experiences } & $\begin{array}{c}\text { Experience was not positive nor negative, different than past and unsure about } \\
\text { their experience }\end{array}$ & General \\
\hline & Dissatisfied with certain aspect of the treatment plan or team & General \\
\hline & $\begin{array}{l}\text { Meeting with the team as a whole was either a new and enjoyable, different or } \\
\text { was similar to past experience }\end{array}$ & General \\
\hline & Hopefulness about treatment plan & General \\
\hline & Improved communication with youth & General \\
\hline & Felt comfortable and supported by team members & General \\
\hline & Experience was positive and expectations were better or similar than expected & Typical \\
\hline & Identified and more knowledgeable about pain & Typical \\
\hline & Expectations of treatment plan and team & Typical \\
\hline & Logistics of appointment & Typical \\
\hline & Similar to past treatment experience & Variant \\
\hline & $\begin{array}{c}\text { Felt meeting with the team was an overwhelming, awkward, or weird } \\
\text { experience }\end{array}$ & Variant \\
\hline
\end{tabular}

Note. Number of adolescents ( $\mathrm{n}=12$ ), general: applies to 10-12; typical: applies to 7-9; variant applies to 4-6. 
Conversely, some adolescents typically found their prior treatment to be helpful. Adolescents were satisfied due to their providers finding medications or alternative treatments that worked. For instance, "he [previous provider] had the best plan, he had the pain medication and he was trying some different medications with me to see if a lower dosage could work better, so he tried a little bit more with me rather than just saying "There's nothing I can do." Another adolescent noted, "I think I had pretty good faith because when I was going to the physical therapist for a little bit it did really help like my muscles. It was making them stronger so that it wasn't always hurting."

In the variant category, adolescents indicated their previous providers focused more on treatment than on communication. Adolescents discussed how the providers went straight to treating their pain and did not provide them with sufficient information about their condition. Adolescents also variantly discussed how past providers only dealt with certain aspects of their pain. Adolescents discussed how providers would treat other medical concerns while not attending to their pain or not treating each area of their pain.

\subsection{Expectation of Multidisciplinary Treatment}

Adolescents generally felt hopeful about the multidisciplinary team's treatment plan prior to the initial appointment. Adolescents were hopeful the team would be able to treat and eliminate their pain through the use of medications or other forms of treatments. Other adolescents perceived their pain would stop and the treatment plan would work well. Moreover, some adolescents noted looking forward to dealing with school concerns as part of their treatment plan. One adolescent stated, "I was hoping they would like do something, like put me on something or do something that would make the pain go away." Another adolescent exclaimed, "I hoped I'm gonna get cured and that I would like stop having headaches."

Alternatively, adolescents typically were apprehensive about the unknowns in their treatment plans, such as not being provided with enough information about their treatment, not knowing if the multidisciplinary team would be helpful, whether their treatment would work and what treatment would be provided, or if all their pain would be treated. One adolescent reported, "I was anxious to go into the meeting to see what their input was and how they were or what they were going to do about it." Another adolescent stated, "I was a little intimidated. I was apprehensive I guess. I didn't really know, like, what they were going to do."

Additionally, as part of their treatment plan, adolescents typically predicted they would receive medication. Feelings were mixed with some adolescents hoping they would receive new medication, while others were concerned about being prescribed additional medication. One adolescent stated, "I knew or I hoped that they would give me some kind of medication to finally get my headache away 'cuz I've been living with it for a year and a half." Conversely, another adolescent reported, "I was worried that I was gonna be put on more medications that I'm already on."

To a lesser extent, and noted as a variant category, adolescents predicted a negative view of treatment; such as, they would not do anything different or be able to help.

\subsection{Expectations of Multidisciplinary Team}

Adolescents typically felt neutral (e.g., OK) or did not identify any feelings or expectations prior to meeting the multidisciplinary team for the first time. For instance, one adolescent explained, "I felt ok, because I had already met one of them in the hospital." While another adolescent reported, "I didn't really have many expectations." However, adolescents typically expected to communicate with the multidisciplinary team about their pain. For example, one adolescent stated, "I thought it would be like one of those things where they would be like all touchy and all talking." Another adolescent reported, "I just thought they was goin' to talk to me about my back again."

To a lesser extent, and noted as a variant category, adolescents did not know what to expect from or knew little about the multidisciplinary team appointment. Adolescents noted they did not know what treatment they would provide or what questions they would ask. Adolescents also variantly expected a visit similar to other providers they have seen in the past. Adolescents discussed how they predicted the multidisciplinary team would treat them in a similar way as did the previous providers. Finally, adolescents variantly discussed their expectation of seeing multiple doctors and other team members as well as a prediction about the length of the appointment.

\subsection{Family Involvement}

Generally, adolescents reported their parents or guardians led the communication with the multidisciplinary team members. Parents generally asked and answered questions, took care of setting up the appointments, and managed their treatment plan. One adolescent stated, “I didn't really tell them, my mom did anyway. She was the one doing all the talking and I was just sitting there." Another adolescent reported, "She [mom] can go home and explain it [treatment plan] whereas I would probably be more worried about what they're saying."

Adolescents typically explained that their parent or guardian gave support throughout their pain experience. Support was perceived when parents/guardians went to all their appointments, acquired knowledge about their pain, and communicated with youth about their pain. One adolescent reported, "Mom...she would always ask me how I was feeling and what kind of pain number I had to see if it was helping or if it was making it worse." Another adolescent explained, "She's gone with me to all my appointments and she knows. And so it's nice to have someone sitting there with you hearing it [information]."

Adolescents also typically reported their 
parents/guardians were hopeful about the multidisciplinary team's treatment approach. Adolescents reported their parents/guardians were excited about the treatment plan, and were happy with the team and their communication. One adolescent reported, "We were both more hopeful or it's a step forward and hopefully it will be the one to help." Another adolescent noted, "She feels pretty much the same way I do. She was really excited about the safety net and she puts it as a "rescue plan."

\subsection{General Experience with the Multidisciplinary Treatment Team}

Adolescents generally described their experience with the multidisciplinary team as different than with past providers; yet, neither positive or negative. Some adolescents were even unsure about their experience or did not want to be at the appointment. One adolescent noted, "It was OK. I mean, like, I don't remember every single thing." Another adolescent stated, "There wasn't a positive or a negative because it wasn't really bad or good, it was just an appointment that I didn't want to go to." It was also found that adolescents generally were dissatisfied with certain aspects of their treatment plan or team. Adolescents reported being prescribed too much medication, felt both physical and psychological aspects would be unhelpful in treating their pain, disliked certain team members' approach, did not appreciate activity restrictions, and did not like the emphasis on talking and answering questions. For example, one adolescent stated, "well my thoughts at first, the doctor seemed kinda like...uhh ...I dunno he seemed like he wasn't really like he wanted to do anything like it's not going to get any worse." Another adolescent explained, "The lady that was going to help me with school, kind of talked about going to different places in your head, and I really wasn't fond of that... I really didn't like that."

At the same time, adolescents generally found meeting with all members of the multidisciplinary team simultaneously as either new and enjoyable, or similar to past provider experiences. One adolescent reported, "Oh, it was okay...they - it was a lot of people explaining to me all at the same time, but--It worked out pretty good, but I'm used to only one person talking to me at once-at a certain time and not like everybody talking to me at the same time...Like I have to switch people I'm talking to every so often." Another adolescent stated, "I was expecting like, one doctor to come in and talk to me and then leave and then have another doctor. And that's kind of what happened, but at the beginning they were both there and they were both talking to each other and to me and my mom. So, it was different and it was a lot better."

Additionally, adolescents generally felt hopeful and confident about their treatment plan. Adolescents reported feeling hopeful about the medication, physical therapy, psychotherapy, acupuncture, and school accommodations. One adolescent reported, "I think it was pretty good. They told me in a month I got to do the exercises every day and
I'm supposed to listen to the tape at least once a day. I think that it'll work out, cause if I can get my muscles stronger then have the medicine so that it doesn't always hurt, I can do more and not be in pain all the time." Another adolescent stated, "I kind of was really hoping that it would work...and to see if it helps the pain. I think I had more faith in this one [doctors] than other ones because they gave me more variety of things I could do to maybe get rid of this pain. And more things that I like to do-like I love to swim, and by being able to do that, that really made things easier on me....and by doing exercises there were things I could do right here inside the house."

Adolescents also generally enjoyed the increased communication between them and the multidisciplinary team. Adolescents reported providers told them additional information about their condition and treatment, listened to their story, talked directly to them, and in general, appreciated their style of communication. One adolescent reported, "I definitely like the way they talked to me and explained things. Like I said, the talking to us, explaining, "this is what we are going to do, this is how we are going to do it." Another adolescent reported, "he listened to my story and exactly what was going on in my life."

Adolescents also generally reported feeling comfortable and supported. Adolescents described the team was friendly, understanding, and caring. One adolescent reported, "They were like nice and it kind of help me be more comfortable." Another adolescent stated, "They were extremely nice. Um, they sat there and listened to me and didn't interrupt me at all unless they had a question. And just like, like each one of them like looked like they wanted to be there."

Generally, adolescents felt providers were knowledgeable, able to identify and treat their pain, and provided them with more information about their condition. One adolescent reported, "Yeah, I got to know everything that I wanted to know. I learned some things that I didn't know before, like the social part being a major crucial part." Another adolescent reported, "It was, I think the Pain Clinic did better...I'm glad they found out 'cause they found out something. Well, they told me what was causing the pain "cause I went in after they fixed it."

Typically, adolescents reported their expectations were either met or exceeded when meeting with the multidisciplinary team. Adolescents noted it was a positive experience and were satisfied with their treatment plan. For example, one adolescent reported, "It was similar to what I wanted because umm I kind of wanted some exercises or things like that to do or more of a range of things that I could do to try to get rid of this pain." While another adolescent stated, "Different but better, [expectations] I thought they did pretty good. They, it was...It was about, it was a little better than the doctor I have now."

On the other hand, adolescents typically noted their expectations for the treatment plan and team were not met because they did not agree with the treatment plan; were not different than expected or did not hold any expectations. 
They also noted their expectations were similar to what they expected, yet, different due to the psychological component to treatment. For example, one adolescent reported, "Umm. The part not met was probably when he said that he didn't really think I needed like that much like stuff for my back." While another adolescent noted, "I expected them to give me some medicine to help me and they did." Adolescents also typically noted their logistics of the appointment; such as, meeting with certain team members, organization in which they met team members, length of the meeting, and the location of the meeting. For example, one adolescent stated, "It was just all in one room, everybody was in there." While another adolescent noted, "They were a lot more detailed than a lot of other doctors. My appointment was much longer and more extensive than any of my other appointments."

Adolescents variantly reported their meeting with the multidisciplinary team was similar to their past treatment experiences. They discussed how previous teams asked the same questions, how they held a similar level of hopefulness for both treatment teams, received similar treatments, communication, and disappointments. Adolescents also variantly noted their meeting with the team was an overwhelming, awkward, nerve racking or a weird experience.

\section{Discussion}

This study contributes to understanding the experiences of adolescents with chronic pain as they encounter their first multidisciplinary team meeting. While the literature has found a multidisciplinary approach to the treatment of chronic pain effective, research focused on understanding the adolescent patient's experience has been neglected. Our findings provide an in-depth understanding of adolescents' expectations prior to treatment, changes in expectations throughout the initial intake, and comparisons with other forms of treatment.

With regard to adolescents' previous experience with their past providers and their treatment approach, results suggest the majority of adolescents felt that the treatment from their previous providers was unhelpful and they were dissatisfied with the care received. Particularly, they were dissatisfied with the medication management. Also noteworthy, but to a lesser extent, was that previous providers took less time to communicate with the youth or only treated certain aspects of their pain. However, the same or another subset of adolescents found their treatment helpful or did not report whether their previous experience was positive or negative because they just noted differences between providers and could not account for their past experiences.

With regard to adolescents' expectations prior to the initial appointment, they were generally hopeful about the treatment plan and felt the team would be able to provide medications or alternative forms of treatment. Adolescents further discussed how their parent or guardian shared their optimism. Yet, the same or a portion of adolescents felt worried or nervous about what would be involved in the treatment plan and whether it would work. When it came specifically to prescribing medications, feelings were mixed with some adolescents hoping they would receive new medication, while others were concerned about being prescribed additional medication. To a lesser extent, some adolescents felt the multidisciplinary treatment team would not be able to do anything different and the treatment plan would be unhelpful.

It was also typical for adolescents to report not holding any expectations, and in fact, some adolescents did not know what to expect when meeting with the multidisciplinary pain team for the first time. However, adolescents felt this experience would provide them with an opportunity to talk about their pain with hope that the treatment plan would be successful. Adolescents explained that generally their parent or guardian accompanied them to the appointment and took a lead role in communicating with the team and providing support around their treatment regimen.

In relation to adolescents' experience during and after their appointment, most found their first experience and expectations of meeting with the multidisciplinary pain team to be neutral, indifferent, or were dissatisfied with certain aspects of the team or their treatment. Adolescents reported unhelpful aspects to their treatment, for example, being prescribed too much medication. This was not an unusual reaction for these adolescents as they were generally dissatisfied with their prior medication management from previous providers; and for some adolescents, these were concerns before going to their first multidisciplinary team appointment and the reason their expectations were not met.

Nevertheless, most adolescents also felt providers were knowledgeable, and therefore able to identify and treat their pain which may have influenced their level of hopefulness and confidence around their treatment plan. Even though the treatment plan included medications and other forms of treatment, adolescents were generally hopeful. Some adolescents also reported a positive experience during their first meeting and their expectations were even better or similar to what they expected. Regardless, nearly all adolescents appreciated the "team" aspect of the multidisciplinary approach to treatment. Specifically, adolescents reported the team had instilled hope, while appreciating the comfort, support and direct communication.

The seemingly contradictory reports from the adolescents become understandable when the complexities of their experiences are taken into account. The transition from general to specialized care can create excitement, hope, and confidence with a new eye on delivery of services, while at the same time, feelings of indifference or disappointment may arise with the realization that new and improved methods of treatment may not be forthcoming. Variability in responses regarding satisfaction with the initial appointment may be attributable to the type of past experiences, number of previous practitioners, and adolescents' ability to process and scrutinize multiple treatment options. Taken together, the adolescents' experience is a blend of positive and 
negative perceptions. To the extent that they process the information provided during the initial appointment, the adolescents report positive experiences (e.g., feeling hopeful, enjoying the opportunity to meet all team members, enjoying the increased communication)as well as negative experiences (e.g., certain aspects of the appointments and the outcome such as disappointment over medications, emphasis on psychological issues, a team member's interaction style).

The health implications of this research could be far reaching. By examining the first hand experiences of adolescents receiving a multidisciplinary treatment for their pain, health care providers may have a better understanding of how to communicate and intervene with different types of interventions and approaches; thus lessening the risk of serious health consequences and improving quality of life.

Results from this study may suggest several ways to enhance communication and interventions when evaluating adolescents and their families for their first appointment. Given that the majority of adolescents were dissatisfied with their previous medical care, it is important to fully listen to their narratives and belief systems in order to determine the gaps in their past treatment history. This may provide practitioners with the opportunity to remediate and address their concerns in a thoughtful way. Furthermore, the adolescent and family members may benefit from an exploration of beliefs and education around medication management. Finally, since adolescents appreciated the direct communication it may be important to continue to engage these youth, while also providing a focus on the provision of hope and success of the treatment plan.

There were several limitations to the current study. The sample only included a small number of participants. Part of the interview protocol was semi- structured, asking the adolescents to recall specific instances of their encounters rather than having them evoke the events on their own. Therefore, the possibility exists that all adolescents' reports may be subject to recall bias.

\section{Conclusions}

This study showed that adolescents with chronic pain are generally hopeful about their first appointment with a multidisciplinary care team. For both patients and parents, the typically extensive, and disappointing past medical history doesn't diminish hope that the team will be able to provide answers and improved treatment. Adolescents also have concerns and fears over aspects of treatment, such as medications. Satisfaction is generally better when given opportunities to be fully engaged. Future investigations of treatment from the patient's perspective are warranted.

\section{REFERENCES}

[1] Greco, C. D. (2003). Management of adolescent chronic pelvic pain from endometriosis: A pain center perspective. Journal of Pediatric and Adolescent Gynecology, 16, S17-19.

[2] Magnusson, J. E., Riess, C. M., \& Becker, W. J. (2004). Effectiveness of a multidisciplinary treatment program for chronic daily headache. The Canadian Journal of Neurological Sciences, 31, 1, 72-79.

[3] Jensen, M. P., Turner, J. A., \& Romano, J. M. (1994). Correlates of improvement in multidisciplinary treatment of chronic pain. Journal of Consulting and Clinical Psychology, $62,1,172-179$.

[4] Hechler, T., Martin, A., Blankenburg, M., Schroeder, S., Kosfelder, J., Hölscher, L., Denecke, H., Zernikow, B. (2011). Specialized multimodal outpatient treatment for children with chronic pain: Treatment pathways and long-term outcome. European Journal of Pain,15,976-984.

[5] De Ble'Court, A. C. E., SchiphorstPreuper H. R., Van Der Schans C. P., Groothoff J. W. \&Reneman M. F. (2008). Preliminary evaluation of a multidisciplinary pain management program for children and adolescents with chronic musculoskeletal pain. Disability and Rehabilitation, $30,1,13-20$.

[6] Hill, C. E., Thompson, B. J., \& Williams, E. N. (1997). A guide to conducting consensual qualitative research. The Counseling Psychologist, 25, 4, 517-572.

[7] Hill, C. E., Thompson, B. J., Hess, S. A., Knox, S., Williams, E. N., \&Ladany, N. (2005). Consensual qualitative research: An update. Journal of Counseling Psychology, 52, 2, 196-205.

[8] Schlosser, L. Z., Hill, C. E., Knox, S., \&Moskovitz A. R. (2003). A qualitative examination of graduate advising relationships: The advisee perspective. Journal of Counseling Psychology, 50, 2, 178-188. 